\title{
Producción de café (Coffea arabica L.) en respuesta al manejo específico de la fertilidad del suelo
}

\section{Production of coffee (Coffea arabica L.) in response to the specific handling of soil fertility}

\section{Produção de café (Coffea arabica L.) em resposta a gestão específica da fertilidade do solo}

\author{
Luz Adriana Lince Salazar ${ }^{1} \&$ Siavosh Sadeghian Khalajabadi² \\ ${ }^{1}$ Geóloga, Estudiante de maestría en Desarrollo Sostenible y Medio Ambiente en la Universidad de \\ Manizales, Colombia. ${ }^{2}$ Ingeniero Agrónomo, Magister en Ciencias Agrarias énfasis Suelos, \\ Doctor en Ciencias Agropecuarias énfasis Fisiología Vegetal. \\ ${ }^{1,2}$ Centro Nacional de Investigaciones de Café- CENICAFE. Manizales. Colombia. \\ 1'luz.lince@cafedecolombia.com, 2Siavosh.Sadeghian@cafedecolombia.com
}

\section{Resumen}

El estudio de la variabilidad espacial es el principal insumo para el manejo específico por sitio-MES, con fines de optimizar los recursos, al reducir los costos de producción de los cultivos y el impacto negativo sobre el ambiente. Con el objetivo de determinar el efecto del MES en la producción del café, durante los años 2011 y 2012 se realizó un experimento del manejo de la fertilidad del suelo en café (Coffea arabica L.), Variedad Castillo® en lotes con áreas diferentes (1,0, 2,0 y 3,5 ha), ubicados en tres fincas del departamento del Quindío, Colombia. Se evaluó el efecto de tres tratamientos en la producción de café cereza: i) testigo sin fertilización; ii) manejo de acuerdo a los resultados de los análisis de suelo, logrados mediante un muestreo convencional en zigzag y, iii) manejo por sitio específico conforme lo indicaron las áreas homogéneas, obtenidas mediante mapas de variabilidad espacial. En ninguno de los lotes hubo efecto de los tratamientos, respuesta que se relacionó con uno o más de los siguientes factores: tiempo relativamen- te corto de evaluación, fertilidad del suelo, reservas de los nutrientes en las plantas, y el número reducido de repeticiones (tres por tratamiento).

Palabras clave: Coffea arabica L., fertilización, variabilidad espacial, geoestadística

\section{Abstract}

The study of variability it is the main input for sitespecific management (SSM), has the objective of optimizing resources by reducing crops production costs and negative impact on the environment. In order to determine the effect to SSM in coffee production, during the years 2011 and 2012, a study of soil fertility management in coffee (Coffea arabica L.) Castillo® variety was undertaken. The experiment was developed in three plots of land of different areas (1.0, 2.0, and 3.5 has), located on three farms in the department of Quindío, Colombia. The effect of three treatments of fertilization in coffee cherry production was evaluated: (i) blank sample without 
fertilization; (ii) management according to the results of the analysis of soil, using conventional sampling, and (iii) site-specific management according to homogeneous areas, obtained by spatial variability maps. However, effects of the treatments were not observed in none of the plots of land. This response was related with one or more of the following factors: the relatively short time of evaluation, soil fertility, reserves of nutrients in the plants, and the small number of repetitions (three per treatment).

Key-words Coffea arabica L., fertilization, spatial variability, geostatistical

\section{Resumo}

O estudo da variabilidade especial é o principal insumo para o manejo específico por sitio-MES, sendo feito para otimizar os recursos ao reduzir os custos de produção das culturas e o impato negativo sobre o ambiente. $\mathrm{O}$ objetivo desse trabalho foi determinar o efeito de MES na produção de café, variedade Castillo®. Para tanto, foi feito um experimento de manejo da adubação do solo em áreas experimentais diferentes $(1,0,2,0$ y 3,5 ha) localizadas em três rosas do estado de Quindio, Colômbia. Foi avaliado o efeito de três tratamentos na produção de café cereza: i) Testemunha sem adubação, ii) manejo de acordo aos resultados dos análises de solos, obtidos mediante amostragem convencional em "zigzag" e, iii) manejo por sitio específico conforme indicado pelas áreas homogêneas obtidas mediante mapas de variabilidade espacial. Nenhuma das áreas experimentais presentou efeito dos tratamentos, sendo que essa resposta foi relacionada com um ou mais dos seguintes fatores: tempo relativamente curto de avaliação, adubação do solo, reservas de nutrimentos nas plantas e o número reduzido de repetições (três por tratamento).

Palavras-chave: Coffea arabica L., adubação, variabilidade espacial, geoestatística

\section{Introducción}

La producción de las especies cultivadas cambia a cortas y largas distancias en virtud de la variabilidad del clima, el suelo y el manejo (Blackmoore, Godwin \& Fountas, 2003; Aggelopooulou, Castrignanò, Gemtos \& Benedetto, 2013). En cuanto a este último factor, la fertilización ha sido señalada como la principal causa (de Paul \& Lal, 2014; Barik, Aksakal, Islam, Sari \& Angin, 2014). El conocimiento de la variabilidad espacial es una herramienta con la que se define el manejo por zonas homogéneas, que permite hacer un Manejo Especifico por Sitio-MES (Ferguson, Lark \& Slater, 2003), por medio del cual se optimizan los recursos al reducir los costos (Cid-Garcia, Albornoz, Rios-Solis \& Ortega, 2013) y el impacto negativo sobre el ambiente, lo que lo convierte en una de las estrategias de adaptación al cambio climático (Lal, 2004).
Los procedimientos que comúnmente se emplean para establecer zonas homogéneas son la representación de la variabilidad mediante mapas de contornos y la simulación estimada de la varianza de los valores de la interpolación (Goovaerts, 1998); en este sentido, los aspectos de mayor interés han sido las propiedades del suelo y la producción (Blackmore, 2000; Ferguson et al. 2002; Pedroso, Taylor, Tisseyre, Charnomordic \& Guillaume, 2010; Zhu, Lin \& Doolittle, 2013). Son ejemplo de lo anterior los estudios desarrollados por Moral, Terrón \& Silva (2010), Kweon (2012), Scudiero et al. (2013), Peralta \& Costa (2013) y Mouazen, Alhwaimel, Kuang \& Waine (2014), basados bien sea en el análisis de las propiedades del suelo a nivel de laboratorio, o a través de la evaluación de la conductividad eléctrica medida en campo o mediante cambios geomorfológicos. 
La interpolación se puede realizar por dos vías, la determinística y la geoestadística; esta última se basa en la teoría de regionalización de variables, con el uso de semivarigramas y métodos de predicción o extrapolación, con un nivel de confianza determinado (Wilding \& Drees, 1983). Sus mayores aplicaciones para suelos, están en el mapeo de unidades, el estudio de génesis y el comportamiento de algunas propiedades físicas como límites de Atterberg y distribución de tamaño de partículas, y en menor proporción para la predicción de los nutrientes y la distribución de contaminantes (plaguicidas) (Krasilnikov, Carre \& Montanarella, 2008), debido que para estos casos la semivarianza raramente muestra un modelo idealizado (Campbell, 1978; Burgess \& Webster, 1980; Hattab, Hambli, Motelica-Heino \& Mench, 2013).

Entre los estudios de MES de la fertilidad del suelo se pueden mencionar los realizados por Ortega \& Santibáñez (2007) para el cultivo de maíz en Chile; Calderón, Martínez \& Henao. (2008) para el cultivo del mango en Colombia, Rodríguez, González, Leiva \& Guerrero (2008) para el cultivo de maíz en Colombia; Tesfahunegn, Tamene \& Vlek (2011) para la planificación agrícola, forestal y servicios ambientales en Etiopia; Davatgar, Neishabouri \& Sepaskhah (2012) para el cultivo de arroz en Irán. Pese al incremento constante en el uso del MES durante las últimas décadas, los resultados de algunas investigaciones sugieren que en parcelas pequeñas e irregulares es más compleja su implementación que el manejo convencional (Mouazen, et al. 2014). Al respecto, Cid-García et al. (2013) sostienen que es posible regularizar las áreas de los lotes mediante algoritmos de variabilidad.

Para Espinosa, Mite, Cedeño, Barriga \& Andino (2005) la adopción del MES en los cultivos perennes que se cosechan manualmente, presenta ciertos retos relacionados con el alto costo inicial de la implementación, el desconocimiento de herramientas tecnológicas y la falta de monitoreo mecánico del rendimiento. Dicha situación se asemeja a las condiciones del cultivo de café en Colombia, del que se encuentra poca información al respecto; razón por lo que se realizó la presente investigación con el fin de determinar el efecto del MES en la producción del café a nivel de lote.

\section{Materiales y métodos}

La investigación se realizó entre los años 2011 y 2012, en la región cafetera del departamento del Quindío, en el flanco occidental de la cordillera central de Colombia, en las unidades de suelo Chinchiná y Quindío (Typic Melanudands y Typic Hapludands, respectivamente), según la denominación de la Federación Nacional de Cafeteros.

Se trabajó en tres lotes de café con diferentes áreas (1,0, 2,0 y 3,5 ha), ubicados en las fincas $\mathrm{La}$ Bella (municipio de Calarcá, vereda La Bella), La Herradura (municipio de Armenia, vereda Murillo) y El Cortijo (municipio de Armenia, vereda Mesopotamia), respectivamente. Geomorfológicamente el lote de 1,0 ha estaba conformado por dos subunidades geomorfológicas, una sima plana ubicada en las regiones norte y oeste, y otra zona de ladera o desprendimiento en la región centro occidental; el lote de 2,0 ha por una unidad geomorfológica de ladera con dirección de desprendimiento suroccidental, y el lote de 3,5 ha conformado por tres subunidades, una plana ubicada en la región más oriental, una secuencia de pequeñas laderas y simas producto de drenajes intermitentes, localizados en la región central y, finalmente, una zona de inclinación inferior a los $20^{\circ}$ en la parte más oriental del lote. Los tres lotes estaban plantados con Variedad Castillo ${ }^{\circledR}$, cuyas edades al inicio del experimento fueron de tres años, a plena exposición solar, densidades de siembra de 7700, 6400 y 7000 plantas/ha para los lotes de 1,0, 2,0 y 3,5 ha, respectivamente, y el manejo agronómico recomendado por Cenicafé.

Cada uno de los lotes fue georreferenciado mediante un sistema en retícula de $20 \mathrm{~m}$ x $20 \mathrm{~m}$ y 
en cada punto se tomaron muestras compuestas de suelo (cuatro submuestras por punto) a una profundidad de 0 a $20 \mathrm{~cm}$, adicionalmente, se realizó un muestreo en zigzag para cada uno de los lotes, conformado por 15 submuestras, también tomadas de 0 a $20 \mathrm{~cm}$. Para ambos tipos de muestreo se evaluaron las siguientes propiedades, según las metodologías de análisis descritas por Carrillo (1985): pH, MO, P, K, Mg, Ca y Al.

Para la espacialización de las variables se partió de un análisis exploratorio, enfocado en tendencia central y dispersión, mediante el software Statgraphics; además, se tomó la distribución de las variables en las clases o rangos establecidas por Sadeghian (2008), como criterio para determinar cuáles de ellas requerían de espacialización por contar con representación superior al $10 \%$ en dos o más clases.

En la interpolación se tuvieron en cuenta los métodos geoestadístico y determinístico (IDW inverse distance weighted). Para la aplicación del primero se verificó que el número de pares de datos fuera mayor o igual a 50 (Villatoro, Henriquez \& Sancho, 2007), que se cumpliera con los supuestos geoestadísticos de normalidad, tendencia y estacionariedad, y se calcularon mediante asimetría y curtosis (-2 y 2), curvatura en el plano $X-Y$, y mapas de Voronoi, respectivamente. Posterior a la aplicación del método de interpolación, para obtener las zonas homogéneas los mapas se clasificaron de acuerdo a las categorías establecidas por Sadeghian (2008) para fertilización de cafetales en etapa de producción. El software empleado para la totalidad de este proceso fue ArcMap versión 10.

Con el fin de evaluar el efecto de la fertilización en la producción de café, basado en el estudio de espacialización, en cada lote se seleccionaron tres zonas contrastantes (tres bloques); en cada uno se establecieron tres parcelas de 45 plantas (21 efectivas) que correspondieron a los tratamientos: i) testigo sin fertilización; ii) manejo de N, P, K y cal para la corrección de acidez, de acuerdo a los resultados de los análisis de suelo, logrados mediante un muestreo convencional en zigzag y, iii) manejo por sitio específico, conforme lo indicaron las áreas homogéneas, obtenidas mediante mapas de variabilidad espacial, en el cual se aplicaron dosis de fertilizantes para N, $P$ y K, además de cal para la corrección de acidez. En cada una de las fincas se registró la producción de café cereza durante 20 meses (abril a diciembre de 2011 y enero a diciembre de 2012). Se realizó un análisis de varianza con el fin de evaluar el efecto de los tratamientos en la producción obtenida en cada año y el acumulado. El software empleado para el análisis estadístico de la evaluación de los tratamientos fue Statgraphics Centurion 15.2.

\section{Resultados y discusión}

En el ámbito general se presentó el siguiente orden en el coeficiente de variación para las propiedades del suelo: $\mathrm{P}>\mathrm{Ca}>\mathrm{K}>\mathrm{Mg}>\mathrm{MO}>\mathrm{pH}$ (Tabla 1), resultados que concuerdan con los reportes de Lozano et al. (2004) y Cora \& Bachmeier (2006) y difieren parcialmente de los presentados por Sadeghian (2010), en los cuales K y P representan menor variabilidad que $\mathrm{Mg}$. La menor variabilidad del $\mathrm{pH}$ se puede deber a que éste se mide en escala logarítmica, y por consiguiente se reduce la expresión de la variabilidad; la mayor variabilidad en $P$ se atribuye a la relación que se da entre la aplicación de fertilizantes y la poca movilidad del elemento en el suelo (Roger et al., 2014). 
Tabla 1. Estadísticos descriptivos para las propiedades químicas de los suelos en los lotes evaluados.

\begin{tabular}{|c|c|c|c|c|c|c|}
\hline \multirow{2}{*}{ Estadístico } & $\mathrm{pH}$ & MO & $\mathbf{K}$ & Ca & $\mathrm{Mg}$ & $\mathbf{P}$ \\
\hline & & (\%) & \multicolumn{3}{|c|}{--ー-ー-- (cmolc.kg-1) ------- } & $---(\mathrm{mg} \cdot \mathrm{kg}-1)---$ \\
\hline & \multicolumn{6}{|c|}{ 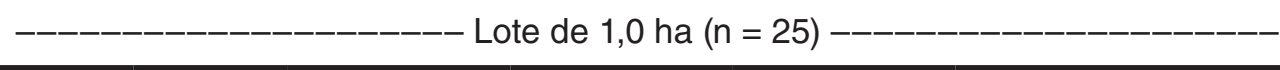 } \\
\hline Mínimo & 4,50 & 5,70 & 0,15 & 0,14 & 0,07 & 36,00 \\
\hline Máximo & 5,20 & 9,40 & 0,52 & 1,94 & 0,40 & 280,00 \\
\hline Media & 4,78 & 7,96 & 0,25 & 0,76 & 0,20 & 93,84 \\
\hline Mediana & 4,80 & 8,20 & 0,23 & 0,61 & 0,19 & 78,00 \\
\hline Moda & 4,80 & 9,40 & 0,20 & 0,37 & 0,19 & 78,00 \\
\hline CV (\%) & 3,00 & 13,00 & 37,00 & 63,00 & 35,00 & 65,00 \\
\hline Asimetría & 0,52 & $-0,70$ & 1,50 & 1,07 & 0,87 & 2,24 \\
\hline \multirow[t]{2}{*}{ Curtosis } & 0,86 & 0,20 & 2,38 & 0,48 & 1,76 & 5,28 \\
\hline & \multicolumn{6}{|c|}{------------------- Lote de 2,0 ha $(n=50)$-------------------- } \\
\hline Mínimo & 4,30 & 4,00 & 0,08 & 0,46 & 0,13 & 5,00 \\
\hline Máximo & 5,80 & 9,20 & 0,72 & 6,55 & 1,95 & 112,00 \\
\hline Media & 5,14 & 6,57 & 0,24 & 2,24 & 0,54 & 27,62 \\
\hline Mediana & 5,20 & 6,45 & 0,21 & 2,00 & 0,47 & 17,50 \\
\hline Moda & 5,20 & 5,90 & 0,26 & 1,05 & 0,45 & 33,00 \\
\hline CV (\%) & 6,00 & 17,00 & 52,00 & 60,00 & 58,00 & 94,00 \\
\hline Asimetría & $-0,38$ & 0,14 & 1,68 & 1,14 & 1,95 & 1,74 \\
\hline \multirow[t]{2}{*}{ Curtosis } & 0,72 & 0,16 & 3,70 & 1,31 & 7,02 & 2,27 \\
\hline & \multicolumn{6}{|c|}{ 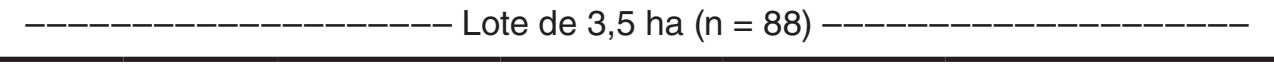 } \\
\hline Mínimo & 4,40 & 5,70 & 0,19 & 0,26 & 0,10 & 4,00 \\
\hline Máximo & 5,90 & 18,10 & 1,22 & 4,31 & 1,18 & 32,00 \\
\hline Media & 5,15 & 13,40 & 0,47 & 1,09 & 0,35 & 11,40 \\
\hline Mediana & 5,10 & 13,60 & 0,41 & 0,91 & 0,32 & 11,00 \\
\hline Moda & 5,10 & 10,90 & 0,34 & 1,03 & 0,30 & 9,00 \\
\hline CV (\%) & 5,00 & 18,00 & 44,00 & 67,00 & 49,00 & 43,00 \\
\hline Asimetría & 0,15 & $-0,44$ & 1,56 & 2,43 & 2,52 & 1,18 \\
\hline Curtosis & 0,84 & 0,53 & 2,99 & 7,14 & 9,73 & 2,64 \\
\hline
\end{tabular}


La categorización de las propiedades, indicó para el lote de 1,0 ha una distribución homogénea de $\mathrm{pH}, \mathrm{P}$, Ca y Mg (Figuras 1, 2, 3 y 4), sugiriendo un manejo generalizado de dichas variables; en contraste, la MO y K (Figuras 5 y 6) presentaron heterogeneidad, por lo tanto se definió un manejo diferencial. En el lote de 2,0 ha, sólo la MO presentó valores en una única clase y en el lote de 3,5 ha, todas las variables presentaron heterogeneidad; por ende, precisaron de manejo diferencial. Los resultados de variabilidad coinciden con lo reportado por Hu, Wang, Li, Huang \& Li (2014), Shi et al. (2014), Barba, Curiel Yuste, Martinez-Vilalta \& Lloret (2013), Molina, Latron,
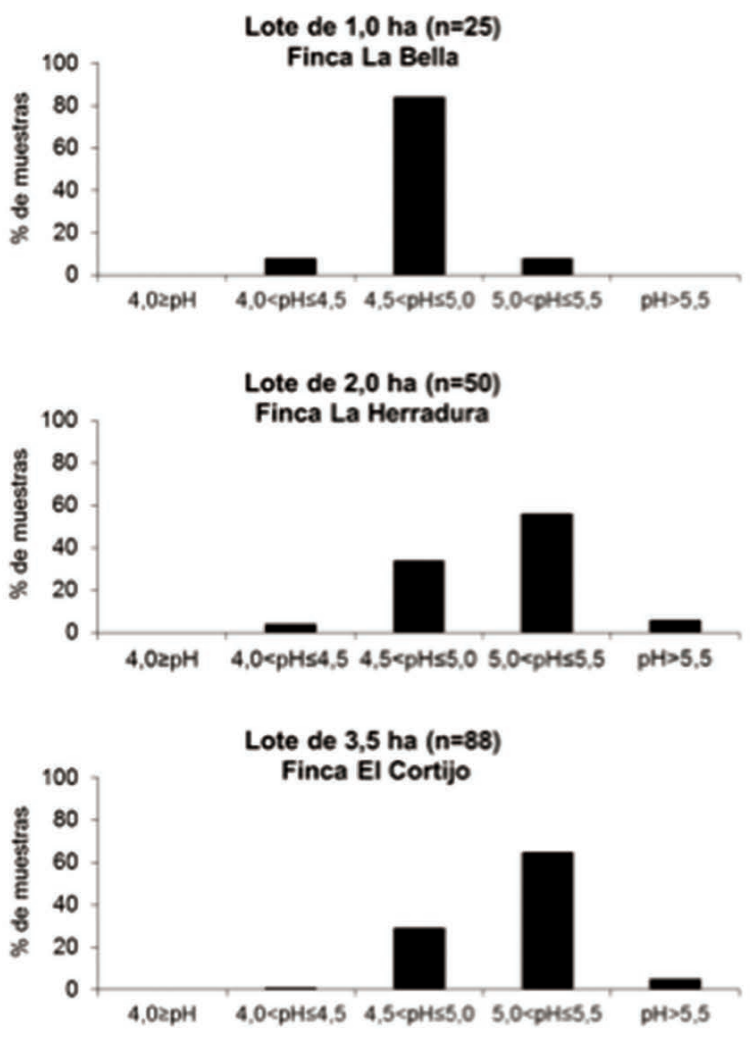

Rangos de $\mathrm{pH}$

Figura 1. Histogramas de frecuencia para $\mathrm{pH}$ en los lotes evaluados.
Rubio, Gallart \& Llorens (2014) y Liu et al. (2015), quienes mencionan que ésta aumenta con el incremento del tamaño del lote, además de verse afecta por el manejo y la topografía; siendo esta última la posible explicación por la cual el lote de 1,0 ha presentó mayor variabilidad de $\mathrm{MO}$ en comparación con el lote de 2,0 ha, ya que en el primero la geomorfología estaba conformada por dos subunidades, una sima plana y una zona de ladera o desprendimiento en el que se reportaron los menores valores, mientras que el lote de 2,0 ha estaba representado por una subunidad geomorfológica conformada por una ladera de pendiente uniforme.
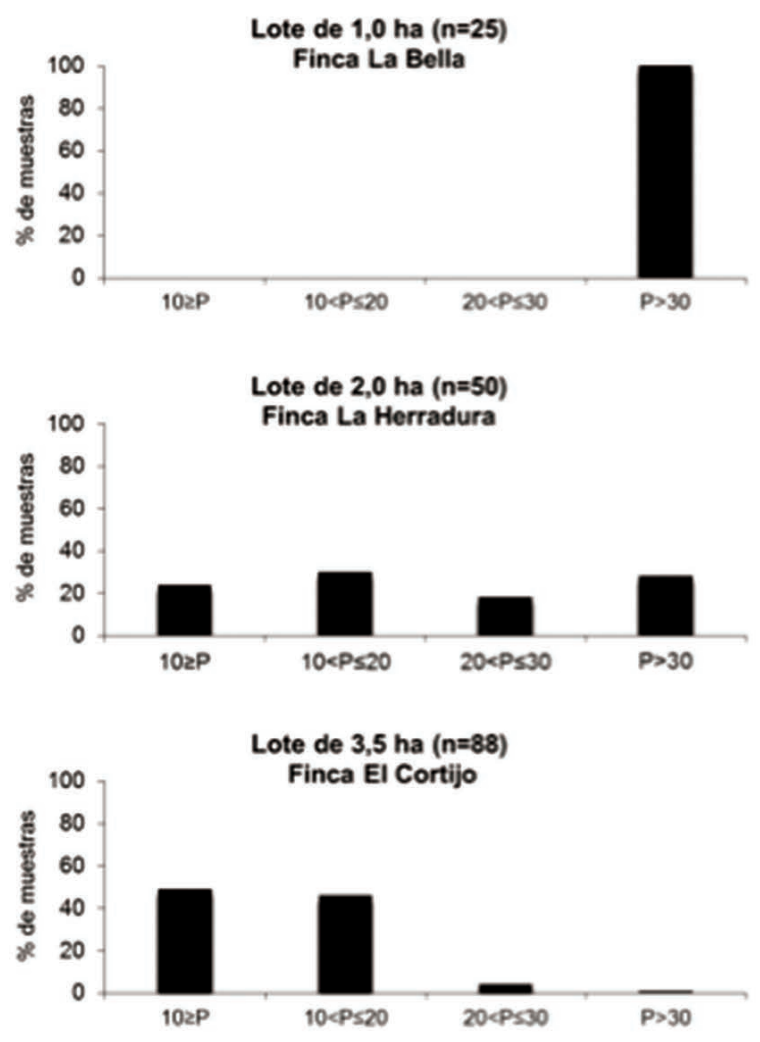

Rangos de $\mathbf{P}$

Figura 2. Histogramas de frecuencia para $\mathrm{P}\left(\mathrm{mg} \cdot \mathrm{kg}^{-1}\right)$ en los lotes evaluados. 

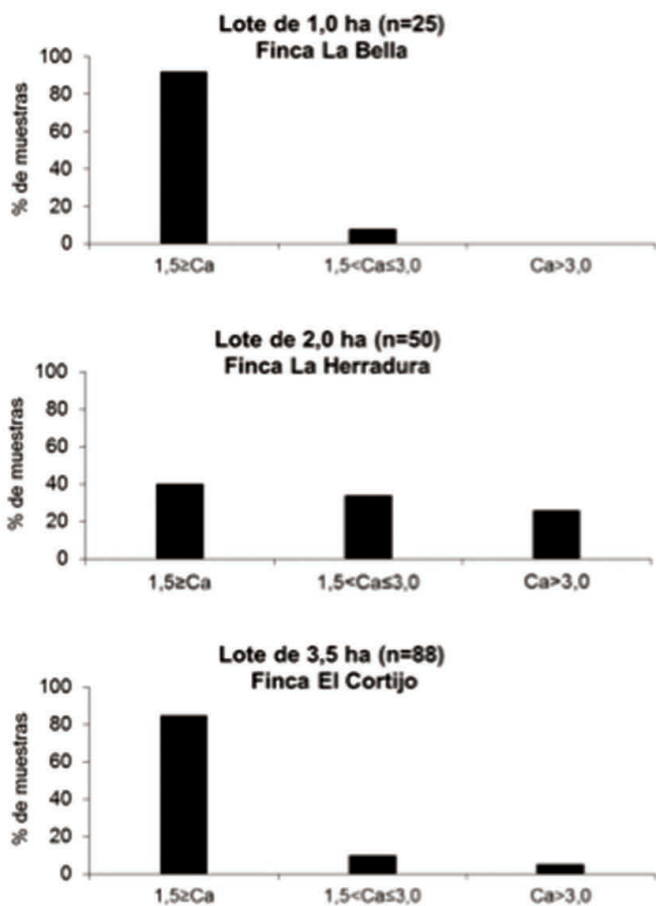

Rangos de Ca

Figura 3. Histogramas de frecuencia para $\mathrm{Ca}\left(\mathrm{cmol}_{\mathrm{c}} \cdot \mathrm{kg}^{-1}\right)$ en los lotes evaluados.
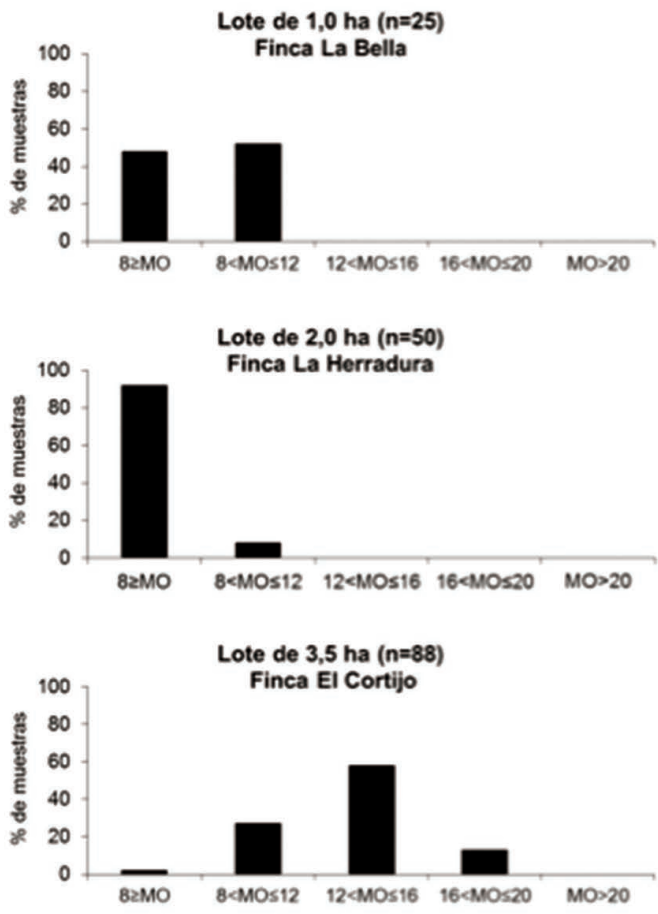

Rangos de materia orgánica

Figura 5. Histogramas de frecuencia para MO (\%) en los lotes evaluados.
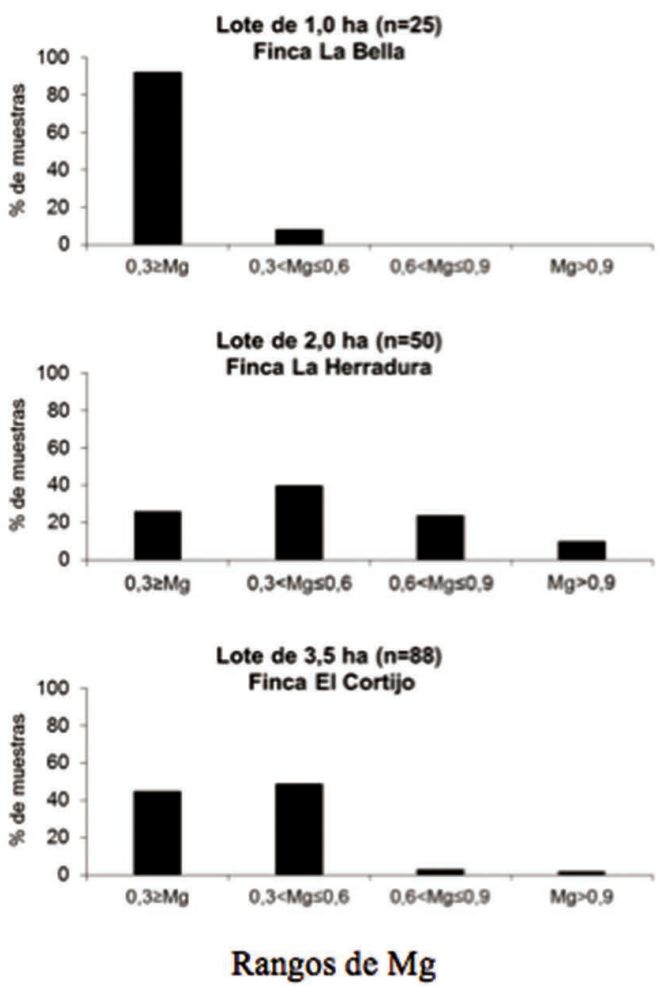

Figura 4. Histogramas de frecuencia para $\mathrm{Mg}\left(\mathrm{cmol}_{\mathrm{c}} \cdot \mathrm{kg}^{-1}\right)$ en los lotes evaluados.
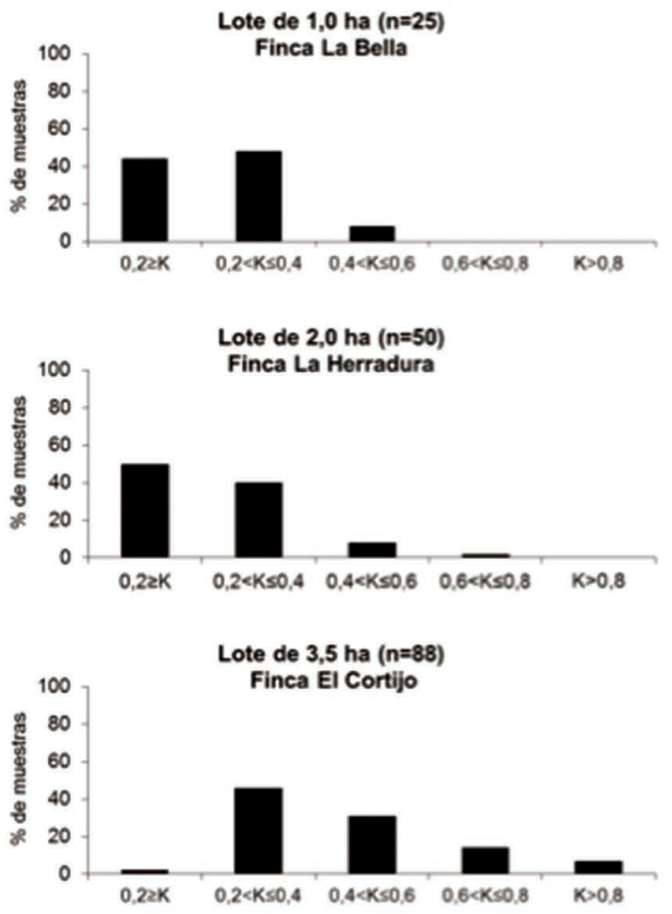

Rangos de K

Figura 6. Histogramas de frecuencia para $\mathrm{K}\left(\mathrm{cmol} \cdot \mathrm{kg}^{-1}\right)$ en los lotes evaluados. 
En el lote de 1,0 ha se aplicó el método IDW para espacializar las propiedades del suelo que requirieron de un manejo diferencial (Tabla 2); esto en razón de que el bajo número de datos analizados $(<50)$ no cumplía con los requisitos del método geoestadístico (Villatoro, Henriquez \& Sancho, 2007). En el lote de 2,0 ha, el pH se espacializó mediante un Kriging ordinario, y las demás propiedades por el método IDW (Figura 3), debido a que no cumplieron con los supuestos de normalidad, estacionariedad y tendencia. En el lote de 3,5 ha, sólo pH y MO cumplieron con el supuesto de normalidad $y$, al igual que las otras, presentaron tendencia en una o ambas coordenadas, en las que el método de transformación que permitió cumplir los supuestos geoestadísticos involucró la interacción de las propiedades con las coordenadas, limitando así la clasificación de los mapas en términos de aplicación de dosis de fertilizante. Una de las posibles razones por las que las variables no cumplieron con los supuestos geoestadísticos es que la distribución natural se ve afectada por el manejo antrópico, representado por la aplicación generalizada e histórica de fertilizantes y enmiendas. Otra causa a considerar es el pequeño tamaño de los lotes seleccionados y, en este caso, el hecho que la distancia de muestreo y el número de muestras fueran reducidos en comparación con otros estudios del mismo tipo.

Tabla 2. Resultados de la interpolación de las propiedades químicas de los suelos en los lotes evaluados

\begin{tabular}{|c|c|c|c|c|}
\hline Propiedad & Método & Modelo & Semieje mayor, $\mathrm{m}$ & RMSE \\
\hline \multicolumn{5}{|c|}{ 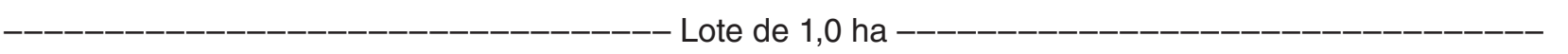 } \\
\hline MO & IDW & $6,429+0,179 x$ & 36,39 & 0,93 \\
\hline K & IDW & $0,139+0,389 x$ & 35,39 & 0,07 \\
\hline $\mathrm{pH}$ & Kriging & $4,811+0,064 x$ & 42,65 & 0,29 \\
\hline$P$ & IDW & $29,637-0,008 x$ & 34,32 & 25,84 \\
\hline K & IDW & $0,169+0,321 x$ & 52,32 & 0,12 \\
\hline $\mathrm{Ca}$ & IDW & $2,320-0,022 x$ & 80,32 & 1,43 \\
\hline $\mathrm{Mg}$ & IDW & $0,459+0,155 x$ & 80,32 & 0,31 \\
\hline $\mathrm{pH}$ & IDW & $4,316+0,158 x$ & 65,71 & 0,23 \\
\hline MO & IDW & $11,371+0,153 x$ & 60,71 & 2,19 \\
\hline$P$ & IDW & $10,555+0,0695 x$ & 109,71 & 4,83 \\
\hline K & IDW & $0,335+0,254 x$ & 50,71 & 0,17 \\
\hline $\mathrm{Ca}$ & IDW & $1,029+0,04554 x$ & 126,71 & 0,72 \\
\hline $\mathrm{Mg}$ & IDW & $0,264+0,18933 x$ & 126,71 & 0,15 \\
\hline
\end{tabular}

IDW: peso inverso de la distancia; RMSE: raíz del error cuadrático medio

Los resultados de espacialización de las variables, y en algunos casos la interpolación por el método coinciden con lo reportado por Rodríguez et al. IDW presenta mejores resultados que el Kriging (2008), al indicar que son igualmente validos los en términos de la correlación con los valores memétodos determinísticos como los geoestadísticos, didos obtenidos en campo (Villatoro et al., 2007; 
Gong, Mattevada \& O'Bryant, 2014). Con respecto a la definición de las zonas homogéneas, los resultados concuerdan con los reportado por varios autores (Ortega \& Santibáñez, 2007; Calderon et al., 2008; Xin-Zong et al., 2009; Silva, Lima, Souza \& Oliveir, 2010; Tesfahunegn et al., 2011; Liu et al., 2014), al indicar que es factible la determinación de zonas con altos y bajos niveles de nutrientes. Sin embargo, hay que tener precauciones al emplear el método IDW, ya que si no se ajustan adecuadamente los parámetros correspondientes al número de vecinos y la distancia de los semiejes, es posible tener islas con efecto "ojo de buey". Por otro lado la marcada tendencia en ambas coordenadas se puede atribuir a patrones geomorfológicos, que según Sigua, Hubbard \& Coleman (2010) han sido poco estudiados en términos de variabilidad de las propiedades del suelo.

En ninguno de los lotes se registró efecto de los tratamientos en la producción de café cereza, ni los dos años (2011 y 2012) ni en el acumulado (Tabla 3). Al respecto, se debe resaltar que los resultados obtenidos pueden no ser del todo concluyentes; de los cuales, la igualdad estadística de los resultados del tratamiento sin fertilización, con los de la aplicación generalizada y el MES, posiblemente se debieron a varios factores no relacionados con el manejo de los tratamientos; entre ellos el número reducido de repeticiones (tres), lo que generó que en varios casos las dosis de los tratamientos del manejo específico y el general fueran similares, (Tabla 4); el tiempo relativamente corto de evaluación, la fertilidad del suelo, las estructuras vegetativas formadas antes del inicio del experimento, y las reservas de los nutrientes en las plantas, debido a que el efecto de la fertilización en la producción de café puede no manifestarse en el primer año en el que se inicia el trabajo de investigación sino al siguiente, ya que el fertilizante suministrado influye principalmente sobre el crecimiento de ese año y la producción del grano tiene lugar en las ramas formadas el año anterior (Mestre, 1977). Resultados similares reportaron Uribe \& Salazar (1981); Uribe (1983) y Sadeghian (2006; 2009) en investigaciones tendientes a evaluar la respuesta de la planta de café a fertilizantes.

Tabla 3. Valores promedio de la producción de café cereza en las parcelas experimentales.

\begin{tabular}{|c|c|c|c|}
\hline \multirow{2}{*}{ Tratamiento } & \multicolumn{3}{|c|}{ Producción café cereza, kg/parcela } \\
\hline & Año 2011 & Año 2012 & Total \\
\hline & \multicolumn{3}{|c|}{ 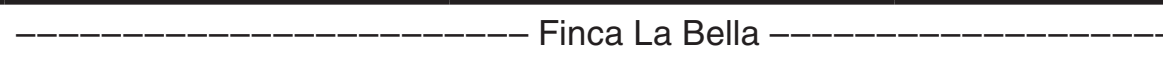 } \\
\hline Sin fertilización & $14,03 \pm 0,59$ & $26,41 \pm 0,75$ & $40,44 \pm 0,66$ \\
\hline Fertilización general & $17,77 \pm 1,03$ & $38,81 \pm 1,15$ & $56,58 \pm 2,17$ \\
\hline \multirow[t]{2}{*}{ Fertilización especifica } & $16,82 \pm 2,12$ & $28,31 \pm 4,44$ & $45,13 \pm 6,38$ \\
\hline & \multicolumn{3}{|c|}{--_-_-_-_-_-_-_-_-_-_-_-_- Finca La Herradura --_-_-_-_-_-_-_- } \\
\hline Sin fertilización & $35,73 \pm 3,38$ & $42,35 \pm 2,81$ & $78,08 \pm 0,75$ \\
\hline Fertilización general & $30,85 \pm 1,06$ & $45,85 \pm 3,36$ & $76,69 \pm 3,09$ \\
\hline \multirow[t]{2}{*}{ Fertilización especifica } & $37,67 \pm 0,97$ & $47,79 \pm 3,26$ & $85,46 \pm 4,19$ \\
\hline & & Ica El Cortijo - & -_-_-_--_- \\
\hline Sin fertilización & $20,29 \pm 2,18$ & $54,26 \pm 7,18$ & $74,55 \pm 7,04$ \\
\hline Fertilización general & $18,45 \pm 2,00$ & $53,44 \pm 8,77$ & $71,89 \pm 6,78$ \\
\hline Fertilización especifica & $18,13 \pm 1,46$ & $49,58 \pm 5,81$ & $67,71 \pm 4,50$ \\
\hline
\end{tabular}

Año 2011: abril-diciembre; Año 2012: enero-diciembre; Total: abril 2011-diciembre 2012.

Las desviaciones corresponden al error estándar. 
Tabla 4. Dosis de fertilizante aplicado en las parcelas experimentales.

\begin{tabular}{|c|c|c|c|c|c|}
\hline \multirow[t]{2}{*}{ Tratamiento } & $\begin{array}{l}\text { Nitrógeno } \\
\text { (N) }\end{array}$ & $\begin{array}{c}\text { Fósforo } \\
\left(\mathrm{P}_{2} \mathrm{O}_{5}\right)\end{array}$ & $\begin{array}{c}\text { Potasio } \\
\left(\mathrm{K}_{2} \mathrm{O}\right)\end{array}$ & $\begin{array}{l}\text { Magnesio } \\
(\mathrm{MgO})\end{array}$ & Cal \\
\hline & \multicolumn{4}{|c|}{ 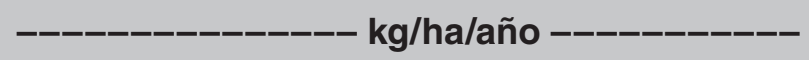 } & kg/ha/2 años \\
\hline & \multicolumn{5}{|c|}{ 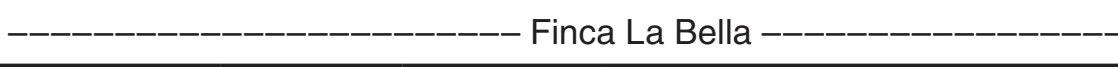 } \\
\hline Fertilización general & 300 & 0 & 260 & 60 & 1000 \\
\hline Fertilización especifica R1 & 280 & 0 & 0 & 60 & 1000 \\
\hline Fertilización especifica R2 & 300 & 0 & 300 & 60 & 1000 \\
\hline \multirow[t]{2}{*}{ Fertilización especifica R3 } & 280 & 0 & 260 & 60 & 1000 \\
\hline & \multicolumn{5}{|c|}{ 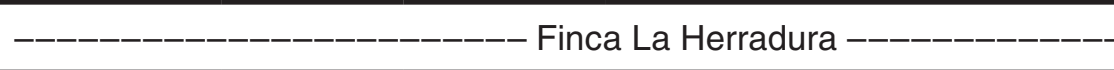 } \\
\hline Fertilización general & 300 & 21 & 260 & 40 & 0 \\
\hline Fertilización especifica R1 & 300 & 21 & 260 & 40 & 0 \\
\hline Fertilización especifica R2 & 300 & 0 & 260 & 15 & 0 \\
\hline \multirow[t]{2}{*}{ Fertilización especifica R3 } & 300 & 21 & 300 & 60 & 0 \\
\hline & \multicolumn{5}{|c|}{ 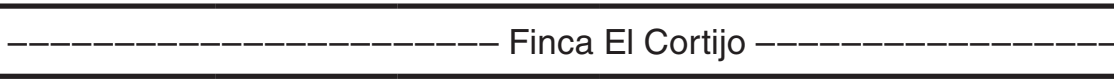 } \\
\hline Fertilización general & 300 & 0 & 260 & 60 & 1000 \\
\hline Fertilización especifica R1 & 260 & 40 & 260 & 60 & 1000 \\
\hline Fertilización especifica R2 & 260 & 60 & 140 & 40 & 400 \\
\hline Fertilización especifica R3 & 260 & 40 & 260 & 60 & 1000 \\
\hline
\end{tabular}

$\mathrm{R}=$ repetición

Por otro lado, sin tener en cuenta el tratamiento sin fertilización y con base en la igualdad estadística de la producción de los tratamientos de manejo general y MES, se considera que es factible realizar un MES, siempre y cuando se justifiquen los costos adicionales; ya que como lo menciona Sadeghian (2008) las aplicaciones del MES para la nutrición del café, cambian según las condiciones del sistema, pues no es lo mismo una explotación mediana o grande en Brasil que en Colombia, conociendo que en éste último más del $80 \%$ de los lotes están por debajo de tamaño de una hectárea, lo que lleva a pensar que muchos productores ya estarían dando un MES a sus cafetales si emplean análisis de suelos.

\section{Conclusiones}

No fue posible determinar el efecto de las diferentes alternativas de manejo en la producción, posiblemente porque el tiempo de evaluación fue corto y el número de repeticiones reducido.

La variabilidad de la fertilidad del suelo puede estar relacionada con el tamaño del lote y los cambios geomorfológicos.

La alta exigencia del método geoestadístico en cuanto a distribución normal, estacionariedad y tendencia de los datos, hace que el método determinístico sea una herramienta eventualmente útil para el establecimiento de zonas homogéneas. 


\section{Agradecimientos}

Los autores expresan sus agradecimientos al Comité Departamental de Cafeteros del Quindío, especialmente a los ingenieros Germán Huertas y Gloria Hincapié, a los colaboradores Anderson Artunduaga y Santiago Arango. A los caficultores propietarios y administradores de las fincas experimentales. A Cenicafé, al investigador científico Hernán Gonzáles, al señor Arturo Gómez y a los colaboradores de la estación experimental Paraguacito.

\section{Literatura citada}

1. Aggelopooulou, K., Castrignanò, A., Gemtos, T. \& Benedetto, D.D. (2013). Delineation of management zones in an apple orchard in Greece using a multivariate approach. Computers and Electronics in Agriculture, 90, 119-130.

2. Barba, J., Curiel Yuste, J., Martinez-Vilalta, J. \& Lloret, F. (2013). Drought-induced tree species replacement is reflected in the spatial variability of soil respiration in a mixed Mediterranean forest. Forest Ecology and Management, 306, 79-87.

3. Barik, K., Aksakal, E.L., Islam, K.R., Sari, S. \& Angin, I. (2014). Spatial variability in soil compaction properties associated with field traffic operations. Catena, 120, 122-133.

4. Blackmore, S. (2000). The interpretation of trends from multiple yield maps. Computers and electronics in agriculture, 26(1), 37-51.

5. Blackmore, S., Godwin, R.J. \& Fountas, S. (2003). The analysis of spatial and temporal trends in yield map data over six years. Biosystems engineering, 84(4), 455-466.

6. Burgess, T.M. \& Webster, R. (1980). Optimal interpolation and isarithmic mapping of soil properties: I. The semivariogram and punctual Kriging. J. Soil Sci., 31:315-331.

7. Calderón, C.A.V., Martínez, L.J.M. \& Henao, R.G. (2008). Variabilidad espacial del suelo y su relación con el rendimiento de mango (Mangifera indica L.). Revista Brasileira de Fruticultura, 1146-1151.

8. Campbell, J.B. (1979). Spatial Variability of soils. Annals of the Association of American Geographers, 69: 544-556.

9. Carrillo P., I.F. (1985). Manual de laboratorio de suelos. Chinchiná, Cenicafé, 111 p.

10. Cid-Garcia, N.M., Albornoz, V., Rios-Solis, Y.A. \& Ortega, R. (2013). Rectangular shape management zone delineation using integer linear programming. Computers and Electronics in Agriculture, 93, 1-9.

11. Cora, A. \& Bachmeier, O. (2006). Número mínimo de muestras necesario para un muestreo edáfico en el Chaco Árido de Córdoba (Argentina). Multequina, 15, 97-102.
12. Davatgar, N., Neishabouri, M. R. \& Sepaskhah, A.R. (2012). Delineation of site specific nutrient management zones for a paddy cultivated area based on soil fertility using fuzzy clustering. Geoderma, 173, 111-118.

13. de Paul Obade, V. \& Lal, R. (2014). Using meta-analyses to assess pedo-variability under different land uses and soil management in central Ohio, USA. Geoderma, 232, 56-68.

14. Espinosa, J., Mite, F., Cedeño, S., Barriga, S. \& Andino, J. (2005). Manejo por Sitio Específico del Cacao Basado en Sistemas de Información Geográfica. INIAP, Pichilingue, Ecuador.

15. Ferguson, R.B., Hergert, G.W., Schepers, J.S., Gotway, C.A., Cahoon, J.E. \& Peterson, T.A. (2002). Site-specific nitrogen management of irrigated maize. Soil Science Society of America Journal, 66(2), 544-553.

16. Ferguson, R.B., Lark, R.M. \& Slater, G.P. (2003). Approaches to management zone definition for use of nitrification inhibitors. Soil Science Society of America Journal, 67(3), 937-947.

17. Goovaerts, P. (1998). Geostatistical tools for characterizing the spatial variability of microbiological and physico-chemical soil properties. Biology and Fertility of soils, 27(4), 315-334.

18. Gong, G., Mattevada, S. \& O'Bryant, S.E. (2014). Comparison of the accuracy of Kriging and IDW interpolations in estimating groundwater arsenic concentrations in Texas. Environmental research, 130, 59-69.

19. Hattab, N., Hambli, R., Motelica-Heino, M. \& Mench, M. (2013). Neural network and Monte Carlo simulation approach to investigate variability of copper concentration in phytoremediated contaminated soils. Journal of environmental management, 129, 134-142.

20. Hu, K., Wang, S., Li, H., Huang, F. \& Li, B. (2014). Spatial scaling effects on variability of soil organic matter and total nitrogen in suburban Beijing. Geoderma, 226, 54-63.

21. Kweon, G. (2012). Delineation of site-specific productivity zones using soil properties and topographic attributes with a fuzzy logic system. Biosystems Engineering, 112(4), 261-277.

22. Krasilnikov, P., Carre, F. \& Montanarella, L. (2008). Soil Geography and Geostatistics. Concepts and Applications. JRC Scientific and Technical Reports, Chapter 1. Office for Official Publications of the European Communities, Luxembourg, pp 204.

23. Lal, R. (2004). Soil carbon sequestration impacts on global climate change and food security. Science, 304(5677), 1623-1627.

24. Liu, Z., Zhou, W., Shen, J., He, P., Lei, Q. \& Liang, G. (2014). A simple assessment on spatial variability of rice yield and selected soil chemical properties of paddy fields in South China. Geoderma, 235, 39-47.

25. Liu, S., An, N., Yang, J., Dong, S., Wang, C. \& Yin, Y. (2015). Prediction of soil organic matter variability associated with different land use types in mountainous landscape in southwestern Yunnan province, China. Catena, 133, 137-144.

26. Lozano, Z., Bravo, C., Ovalles, F., Hernández, R. M., 
Moreno, B., Piñango, L. \& Villanueva, J.G. (2004). Selección de un diseño de muestreo en parcelas experimentales a partir del estudio de la variabilidad espacial de los suelos. Bioagro, 16(1), 61-72.

27. Mestre, A. (1977). Determinación de la rata óptima de fertilización en plantaciones de café sin sombrío. Cenicafé 28(2):51-60.

28. Molina, A. J., Latron, J., Rubio, C. M., Gallart, F. \& Llorens, P. (2014). Spatio-temporal variability of soil water content on the local scale in a Mediterranean mountain area (Vallcebre, North Eastern Spain). How different spatio-temporal scales reflect mean soil water content. Journal of Hydrology.

29. Moral, F.J., Terrón, J.M. \& Silva, J.R. (2010). Delineation of management zones using mobile measurements of soil apparent electrical conductivity and multivariate geostatistical techniques. Soil and Tillage Research, 106(2), 335-343.

30. Mouazen, A.M., Alhwaimel, S.A., Kuang, B. \& Waine, T. (2014). Multiple on-line soil sensors and data fusion approach for delineation of water holding capacity zones for site specific irrigation. Soil and Tillage Research, 143, 95-105.

31. Ortega, R.A. \& Santibáñez, O.A. (2007). Determination of management zones in corn (Zea mays L.) based on soil fertility. Computers and Electronics in Agriculture, 58(1), 49-59.

32. Peralta, N.R. \& Costa, J.L. (2013). Delineation of management zones with soil apparent electrical conductivity to improve nutrient management. Computers and Electronics in Agriculture, 99, 218-226.

33. Rodríguez, J., González, A.M., Leiva, F.R. \& Guerrero, L. (2008). Fertilización por sitio específico en un cultivo de maíz (Zea mays L.) en la Sabana de Bogotá. Agronomía Colombiana, 26(2), 308-321.

34. Roger, A., Libohova, Z., Rossier, N., Joost, S., Maltas, A., Frossard, E. \& Sinaj, S. (2014). Spatial variability of soil phosphorus in the Fribourg canton, Switzerland. Geoderma, 217, 26-36.

35. Pedroso, M., Taylor, J., Tisseyre, B., Charnomordic, B. \& Guillaume, S. (2010). A segmentation algorithm for the delineation of agricultural management zones. Computers and Electronics in Agriculture, 70(1), 199-208.

36. Sadeghian, S., García, J. C. \& Montoya, E. C. (2006). Respuesta del cafeto a la fertilización con N, P, K y Mg en dos fincas del departamento del Quindío. Cenicafé 56(4):58-69.

37. Sadeghian, S. (2008). Fertilidad del suelo y nutrición del café en Colombia. Boletín técnico № 32 Cenicafé.

38. Sadeghian, S. (2009). Calibración de análisis de suelo para N, P, K y Mg en cafetales al sol y bajo semisombra. Cenicafé 60 (1): 7-24.

39. Sadeghian, S. (2010). Evaluación de la fertilidad del suelo para una adecuada nutrición de los cultivos. Caso café. XII Congreso Ecuatoriano de la Ciencia del Suelo, Santo Domingo, Ecuador 17 - 19 noviembre.

40. Scudiero, E., Teatini, P., Corwin, D. L., Deiana, R., Berti, A. \& Morari, F. (2013). Delineation of site-specific management units in a saline region at the Venice Lagoon margin,
Italy, using soil reflectance and apparent electrical conductivity. Computers and Electronics in Agriculture, 99, 54-64.

41. Shi, S.Q., Cao, Q.W., Yao, Y.M., Tang, H.J., Yang, P. Wu, W.B. \& Li, Z.G. (2014). Influence of Climate and Socio-Economic Factors on the Spatio-Temporal Variability of Soil Organic Matter: A Case Study of Central Heilongjiang Province, China. Journal of Integrative Agriculture, 13(7), 1486-1500.

42. Sigua, G.C., Hubbard, R.K. \& Coleman, S.W., (2010). Quantifying phosphorus levels in soils, plants, surface water, and shallow groundwater associated with bahiagrass-based pastures. Environmental Science and PoIlution Research, 17(1), 210-219.

43. Silva, A.F. da., Lima, J.S.S. da., Souza, G.S. \& Oliveira, R.B. (2010). Variabilidade espacial de atributos químicos do solo cultivado com café arábica (Coffea arabica $L$.) sob diferentes manejos. Coffee Science, 5(2), 173-182.

44. Tesfahunegn, G.B., Tamene, L. \& Vlek, P.L. (2011). Catchment-scale spatial variability of soil properties and implications on site-specific soil management in northern Ethiopia. Soil and Tillage Research, 117, 124-139.

45. Uribe, A. \& Mestre, A. (1976). Efecto del nitrógeno, el fósforo y el potasio sobre la producción de café. Cenicafé 27(4):158-173.

46. Uribe, A. (1983). Efecto del fósforo en la producción de café. Cenicafé 34(1):3-15.

47. Uribe, A. \& Salazar, J.N. (1981). Efecto de los elementos menores en la producción de café. Cenicafé 32(4):122-142.

48. Villatoro, M., Henríquez, C. \& Sancho, F. (2007). Comparación de los interpoladores IDW y Kriging en la variación espacial de $\mathrm{pH}, \mathrm{CA}, \mathrm{CICE}$ y $\mathrm{P}$ del suelo. Agronomía Costarricense, 32(1):95-105.

49. Wilding, L.P. \& Drees, L.R. (1983). Spatial variability. In Pedogenesis and soil Taxonomy. I. Concepts and interactions, by L.P. Wilding, N.E. Smeck and G.F. Hall (Editors), $83-116$.

50. Xin-Zhong, W., Guo-Shun, L., Hong-Chao, H., ZhenHai, W., Qing-Hua, L., Xu-Feng, L. \& Yan-Tao, L. (2009). Determination of management zones for a tobacco field based on soil fertility. Computers and Electronics in Agriculture, 65(2), 168-175.

51. Zhu, Q., Lin, H.S. \& Doolittle, J.A. (2013). Functional soil mapping for site-specific soil moisture and crop yield management. Geoderma, 200, 45-54.

\section{Conflicto de Intereses}

\section{Los autores declaran no tener ningún conflicto} de intereses
Recibido: 27 de enero de 2015 Aceptado: 02 de marzo de 2015 